\title{
1. Die bisherigen Standpunkte zur Lösung des Problems
}

1.1. Seit dem Bericht des Jesuiten Stribiņš aus dem Jahre 1606 wissen wir, daß die Letten einen Gott der Pferde - deus equorum - Ūsiņ̌s - kennen. Dessen Name taucht jedoch in späteren Jahren sehr selten auf. Offenbar ist Mannhardt bei der Sammlung von Material über die Religion der Balten auf diesen von Stribiņš erwähnten Gott gestoßen und hat sich, um mehr darüber zu erfahren, an den Rigaer Stadtbibliothekar Berkholz mit der Bitte um ergänzende Auskünfte gewandt. Dieser wiederum richtete sich an den damaligen Pastor Auniņš in Dikḷ. Letzterer antwortete, daß er in seiner Gemeinde in der Sache keine Nachrichten habe finden können. Erst nach seiner Versetzung in die Gemeinde Cesvaine sei er auf den Namen Üsinš gestoßen. Das habe sein Interesse geweckt, sich mit dieser Erscheinung näher zu befassen. So habe er im Jahre 1880 in der damaligen einzigen wissenschaftlichen ,Lettisch-Literärischen Gesellschaft" über diese Frage eine Vorlesung gehalten, in der er seine Forschungsergebnisse vorgelegt habe. Sein Referat erschien ein Jahr später im Druck. Auf die Arbeitsergebnisse von Auniņš werden wir im folgenden, unter Berücksichtigung auch der von ihm ausgewerteten Quellen und seiner Methode, mehrfach zurückkommen. Hier ist vorläufig nur das von Auniņš erzielte Ergebnis festzustellen, um es später mit anderen in dieser Frage geäußerten Auffassungen vergleichen zu können und so den derzeitigen Stand der Forschung zur Lösung des Problems Ūsiṇš zu bestimmen.

1.1.1. Auniņš hat eine größere Zahl von ihm selbst in der Gemeinde gehörter und notierter Dainas (Volkslieder) zur Verfügung gestanden, in denen Ūsinšs erwähnt wird und die einen Einblick in dessen Funktionen, Attribute und Kult gewähren. Auniņš hat auch den Bericht des bereits erwähnten Stribiņš vom Jahre 1606 über Beobachtungen auf einer Missionsreise in Ostlettland an die Leitung des Jesuitenkollegiums in Riga ausgewertet. Ebenso beruft er sich, wie wir sehen werden, auf zugängliches lexikalisches Material. Er hat auch gelegentlich auf Ortsnamen, die in Verbindung mit Ūsiṇš stehen, hingewiesen. Auch der Bericht seines Vorgängers in der Gemeinde Cesvaine über Ūsiṇš hat hierbei eine gewisse Bedeutung gehabt. 
1.1.2. Auniņ̌š Methode ist die historisch-vergleichende. Man darf nicht vergessen, daß er seine Arbeit in den $80 \mathrm{er}$ Jahren des vorigen Jahrhunderts geschrieben hat, weshalb man bei Auniņ̌s nur sehr bedingt von dem Vorhandensein eines besonderen methodischen Bewußtseins sprechen kann. Doch hat er sich bemüht, alle zugänglichen geschichtlichen Daten anzugeben, außerdem analysiert er sie in ihrem genetischen Zusammenhang. Daneben ist noch ein weiterer deutlicher methodischer Zug zu erkennen. Ihm war die damals in ihren Anfängen steckende vergleichende Sprachwissenschaft nicht fremd, wie das sein wiederholtes Berufen auf die bedeutendsten Vertreter dieses Faches in der damaligen Zeit zeigt. Auch hat Auniņš die Forderung, seiner Methode zu folgen, offen ausgesprochen: ,Wie die neuere Sprachwissenschaft sich der vergleichenden Methode bedient, so muß sich die Mythologie auch derselben Methode bedienen."1 „Die Vorstellung des UhBing als eines Pferdepatrons ist offenbar nur die letzte Phase in der Entwickelung des UhBing-Mythus. Die uns vorgelegte Frage: „Wer ist Uhßing?" wird erst dann völlig befriedigend beantwortet sein, wenn wir vorher eine Antwort auf folgende Fragen werden gefunden haben:

1) Ist der UhBing zu allen Zeiten nur einem Theile der Letten bekannt gewesen, oder haben ihn vielleicht in der Urzeit sämmtliche Letten gekannt und verehrt?

2) Von welcher Wurzel stammt der Name des UhBing und was ist nach der Etymologie des Namens die Grundbedeutung desselben?

3) Welches sind die Beziehungen, in denen der heidnische UhBing und der heilige Georg zu einander stehen und läßt sich die Substituirung des letzteren für den ersteren irgend wie erklären?

4) Ist nicht vielleicht im letzten Grunde die ursprüngliche Idee des Uhßing mit der des Lindwurmtödters identisch? Ja, liegt nicht vielleicht dem UhBing-Mythus ursprünglich dieselbe Idee zu Grunde, wie dem Mythus von Siegfried und Baldur bei den Germanen, von Daschdbog, Swaroschitsch und Swantewit bei den Slawen, von Herakles und Apollo bei den Griechen, von Krischna (dem Úberwinder des Drachen Kaliaga), bei den Indern, von Ormuzd bei den Persern etc. Die genügende Beantwortung bevorstehender Frage muß den Männern von Fach vorbehalten bleiben."2

Diese Worte von Auniņš zeigen, daß er sich der Schwierigkeiten und methodologischen Forderungen bewußt war, die mit dem Problem des Ūsinšs verbunden sind. Daher hat er auch darauf hingewiesen, daß die Lösung des Problems späteren Zeiten und Fachleuten überlassen werden müsse. Seine Forschungsresultate sieht er als Vorarbeiten an. Worum handelt es sich?

\footnotetext{
1 Auniņš 1913, 29.

2 Auniṇš 1881, $36 \mathrm{~s}$.
} 
1.1.3. Seine Erkenntnisse hat er wie folgt zum Ausdruck gebracht: „Die Betrachtung der Etymologie des Namens 'Uhßing' hat uns zu der Annahme geführt, daß die ursprüngliche Bedeutung des Uhßing die eines Lichtgottes gewesen sein muß. Sehen wir aber auch ganz von dem Namen ab und betrachten wir nur das Uhßing-Opfer, so weist uns auch dieses auf einen Licht- und Frühlingsgott hin. Der Hahn ist ein Sinnbild des Tageslichtes, die Eier sind nach uralter und weitverbreiteter Symbolik Sinnbilder der lebenschaffenden Naturkraft und Fruchtbarkeit.

$\mathrm{Zu}$ den beiden Zeugnissen des Uhßing-Namens und des UhBing-Opfers kommen nun noch hinzu die Schlüsse, die wir aus den mitgetheilten UhßingLiedern in Betreff der Grundidee des Uhßing ziehen dürfen. Sollen wir alle die in diesen Liedern vorkommenden Prädicate unter Ein gemeinsames Subjekt subsumiren, so kann dies Subjekt nur ein Gott des Lichtes sein, dessen Söhne Morgenrot und Abendrot, ja, Sonne und Mond sind, der die ganze Welt erwärmt, der im Frühling mächtig dahergesprengt kommt auf einem Roße von Stein, Schnee und Eis zermalmend, - der Wälder und Wiesen mit herrlichem Grün bekleidet und nicht blos den Pferden, sondern allen lebenden Wesen die zur Lebenserhaltung nötige Nahrung schafft und spendet. Dieses halten wir für die ursprüngliche Idee des Uhßing. Die ursprünglich großartige, tiefe und umfassende Bedeutung des Uhßing ist allmählig im Laufe der Zeit zu der Gestalt eines Pferde-Patrons (eines sirgubarotajs) verblaßt. Aber eine Ahnung von der ursprünglichen grandiosen Bedeutung hat sich doch noch unter einem Teil des lettischen Volkes bis auf den heutigen Tag erhalten."

Auniņ̌ hat mit diesen Worten einfühlsam und bildhaft das Resultat seiner Forschungen dargelegt. Kürzer und genauer ausgedrückt: Auniņ̌s hat zwei Dinge erkannt, erstens, daß Üsinšs ein Gott des Lichtes ist, und zweitens, daß er , allmählig im Laufe der Zeit zu der Gestalt eines Pferdepatrons (zirgubarotäjs) verblaßt" ist.

Auniņš' Forschungen riefen eine weite und scharfe Polemik hervor, mit der wir uns später eingehender beschäftigen werden. Hier ist jedoch schon zu bemerken, daß er seine im Jahre 1881 geäußerte These von Usiṇš als einem Gott des Lichtes später niemals geändert hat. So schreibt er nochmals im Jahre 1905: ,,[...] ursprünglich ist der Uhsin eine Lichtgottheit gewesen, wie noch Vieles in der Überlieferung diese Behauptung bestätigt, dann ist er in weiterer Entwicklung zu einem Pferdepatron herabgesunken, von dem zuletzt noch Manches ausgesagt wird, was auch von den Hausgeistern berichtet wird. Aber auch auf dieser Stufe verliert er nicht völlig seinen ursprünglichen Charakter."

\footnotetext{
$1 \mathrm{Ib} .41 \mathrm{~s}$.

2 Auniṇš 1905, 29.
} 
1.2. Im Verlauf der Polemik wurde Auninš die Frage gestellt, wie es denn, ausgehend von seiner Ansicht, daß Ūsinšs eine Lichtgottheit sei, erklärt werden könne, daß Ūsiņš auch als Bienenpatron bekannt sei ${ }^{1}$, wie das bereits in der Lexikographie des 18. Jhs. bestätigt werde. Auniņš weist auf die Texte seiner Sammlung (36) hin und bemerkt, daß Ūsiņšs sekundär auch zum Bienengott werden konnte, ebenso wie er sekundär zum Pferdegott geworden war, weil er nämlich mit Frühlingslicht und -wärme das für die Pferde wichtige ",grüne Gras" und die Weide zur Nachtzeit sowie den Bienen ,schöne Knospen und Blumen brachte ${ }^{62}$. Im Jahre 1913, dem Jahr vor seinem Tode, vermochte er noch zu bekräftigen, daß er weiterhin von seiner bereits 1881 geäußerten Ansicht überzeugt sei, Ūsiņš sei eine Lichtgottheit. Wie wir bereits sagten, hat er versucht, seinen Standpunkt entsprechend der vergleichenden Methode seiner Zeit durch Material aus anderen Religionen zu begründen sowie auch Ūsiņš — wie er sich ausdrückt im größeren mythischen Zusammenhang zu sehen: „,Das uns vorliegende Material über den Uhsin-Mythus darf nicht isoliert betrachtet werden, sondern im Zusammenhang mit all dem übrigen, uns überlieferten und von anerkannten Forschern gedeuteten Material über die lettischen Sonnenmythen."3

1.2.1. Endzelins hat sich zuerst im Jahre 1902 kurz über die Etymologie des Namens $\bar{U} \operatorname{sincš~geäußert.~Er~bezweifelte~die~Behauptungen~von~Auniṇš:~}$ ,Nun ist -iňš aber nur ein Deminutivsuffix, und da $\bar{U} \operatorname{siņš~kein~Deminutiv~}$ ist, so halte ich diesen Namen für ein Lehnwort aus dem Germanischen (vgl. ahd. N. Pl. hüsing $\bar{a}$ 'penates', afries. hüsing 'Hausmann'). "4 Dazu hat Endzelīns noch ein paar religionswissenschaftliche Bemerkungen gemacht, nämalich, wenn der Lichtgott zu einem Pferdepatron geworden wäre, sei dieser Gott ,stark degradiert worden". Aber er bemerkt auch, „doch wäre das nicht unmöglich“. Dagegen, ,daß ein Hausgeist sich zu einem Schutzpatron der Pferde entwickeln konnte, ist ja leicht denkbar ${ }^{c_{5}}$.

Bald darauf, im Jahre 1903, veröffentlichte Endzelins einen längeren Beitrag, in dem er zu den vorhin geäußerten Gedanken zurückkehrte und sie noch weiter zu begründen versuchte. Die Ansicht von Auniṇš, Ūsiņ̌s sei ein lettischer Gott, wies er streng zurück. Sein Hauptmotiv war, daß der Name $\bar{U} \operatorname{siņš~,vielleicht~gar~kein~echtes~lettisches~Wort~ist“.~Er~be-~}$ gründet es damit, daß $\bar{U} \operatorname{sinšs~,in~Wirklichkeit~gar~kein~Deminutiv~war,~}$

1 Cf. Endzelīns 1908, 135.

2 Auniṇš 1913, 18.

3 Ib. 27.

1 Endzelins 1902, 330.

5 Ib. Diese Überlegungen von Endzelins sind allgemeiner Natur und zeigen, daB er sich zu Problemen der Religionsgeschichte geäußert hat, die ihm fremd waren. 
sondern die ursprüngliche Wortform. Sonst jedoch sind alle lettischen Wörter mit der Endung -inss Deminutive. "1 Auf diese Weise hat er auch gar keine Schwierigkeiten mit dem in Auniņš' Text vorkommenden Grundwort $\bar{U} s s$ (33), das ,als Abstrahierung aus dem scheinbaren Deminutiv Ūsiňš angesehen werden $m u B^{c}$. So sucht er, indem er die lettische Herkunft des Namens ablehnt, nach einer anderen Erklärung für das Wesen von Ũsinšs. Eine solche hat er bei Grimm gefunden: „Was die Römer mit lar und penas bezeichneten, nannte man in unserer (d. h.: der deutschen) alten Sprache hûsing (hüsing gesprochen) oder stetigot." Er selbst sagt: „Wenn dieses altdeutsche hûsing ('Hausgeist', vom Wort hîs=neudeutsch 'Haus') zusammen mit dem Christengott in unsere Heimat eingeführt wurde, mußte er sich in lettischer Zunge in $\bar{U} \sin , s ̌$ verwandeln."2 Es konnte Endzelins nicht verborgen bleiben, daß diese Erklärung problematisch ist, denn für das deutsche hûsing wäre in lettischer Sprache ein Wort mit stimmhaftem -s-, in lettischer Schreibweise $\bar{U}_{z i n . s}$, zu erwarten gewesen. Diese Form ist auch in Texten der Folklore anzutreffen. Endzelins erklärt sie so, daß ,die in

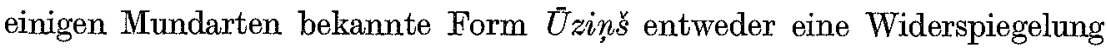
der späteren deutschen Aussprache oder eine von den Letten selbst vorgenommene Veränderung darstellt ${ }^{\varsigma_{3}}$. Nachdem er auf diese Weise die Herkunft des lettischen Namens erklärt hat, bestimmt er in Übereinstimmung damit auch das Wesen von Ūsinš. Dieser ist nach deutschem Vorbild ein Hausgeist. Er hat jedoch bemerkt, daß man nicht völlig rekonstruieren könne, wie sich aus dem Hausgeist ein Pferdegott gebildet habe, doch sei das eher möglich, als dessen Umwandlung aus einem Gott des Lichtes. Endzelins beruft sich ferner auf den Hinweis Grimms auf den engen Zusammenhang des Hausgeistes mit der Feuer- oder Herdstelle des Hauses. Daher sei ,es auch leicht zu verstehen, daß manchmal die Hähne für Ūsiņš in der Küche über dem Herd geschlachtet worden seien und das Blut ins Feuer gelaufen sei und daß man von dem am Tage des Usinšs bereiteten Bier zuerst ins Feuer gegossen habe"4. Er erklärt es auch, daß Ūsinšs gerade am 23. April, d. h. am hl. Georgstag, geehrt werde, der in der Volkstradition auch der Tag des Ūsiņs genannt werde, denn an diesem Tage „,wechselte das Gesinde, aber manchmal zogen auch die Hauswirte von einem Haus in ein anderes; es ist denkbar, daß man dann den Hausgeist Ũsin verehrte, damit er für ein gutes Leben im neuen Jahr sorgte ${ }^{\ll 5}$.

Auniņš fiel es nicht schwer, dieser Ansicht Endzelīns' zurückzuweisen,

1 Endzelins 1903, 25s.

2 Ib. Cf. Grimm 413.

8 Ib.

1 Ib. 26.

5 Ib. 27. 
da sie offensichtliche Mängel enthielt. Er wies zunächst darauf hin, daß Endzelins' Behauptung, ,alle lettischen Wörter mit -ings seien Deminutive", falsch sei, denn in der lettischen Sprache gebe es viele Wörter, die weder jetzt noch früher Deminutive gewesen seien und trotzdem die Endung -inňs aufwiesen ${ }^{1}$. Sachlich wandte ex sich besonders gegen die Ansicht, daß hûsing durch die christlichen Missionare bei den Letten eingeführt worden $\operatorname{sei}^{2}$. Auniņš hat den Zusammenhang richtig erkannt, daß die Letten, wie alle Völker, selbst ihre Hausgeister hatten und daß es keine Hinweise darauf gibt, daß sie diese unter dem Einfluß der christlichen Mission in hüsing umbenannt hätten, woraus sich dann später die Bezeichnung Ūsiṇš entwickelt haben könnte. Endzelīns habe eine spekulative Hypothese aufgestellt, die Auniņš ablehnte.

1.2.2. Endzelīns selbst sah bald ein, daß er sich geirrt hatte, und kam einige Jahre später, 1908, mit einer neuen Hypothese. Sie ist der vorhergegangenen völlig entgegengesetzt. Diesmal suchte er die Lösung bei den Russen. Er gab zu, daß hûsing bei den Deutschen sehr selten vorgekommen und bereits früh verschwunden sei. Mit fester Überzeugung sagt er: „, [..] den lettischen Usinsš wird man nicht vom russischen 'У сень' oder 'Овсень' trennen können. "3 Welches sind diesmal seine Argumente?

Zuerst weist er eines der Argumente des Auniņš, daß Ūsiņš von der indoeuropäischen Wurzel aus- abgeleitet sein könnte, zurück ${ }^{4}$. Dieser Ansicht von Auniņš hatte sich schon früher auch Volteris angeschlossen ${ }^{5}$. Sowohl Auniṇš als auch Volteris beriefen sich auf mehrere russische Forscher - Afanasjev, Potebnja und Famincyn ${ }^{6}$. Endzelins lehnte alle ihre

${ }^{1}$ Cf. Auniñ̌s 1905, 4. Das wird später mehrfach auch in lettischen Grammatiken bestätigt, ebenso Endzelins 1951, 313, § 152; Gramatika 1, 117.

2 Nicht ohne Ironie schrieb Auniņš 1905, 14s: „Haben die frommen christlichen Missionare, Mönche und Priester als sie nach Livland kamen, etwa öffentlich und offieiell das Evangelium gepredigt und dann noch nebenbei den eben bekehrten oder noch zu bekehrenden Heiden Privatstunden in der deutschen Mythologie und im deutschen Aberglauben gegeben.

Oder aber haben die eisenbepanzerten. Ritter sich erst mit den Eingeborenen Livlands blutig gerauft, und alsdann vielleicht den Besiegten gelegentlich beim Methbecher gemütliche Geschichten vom Husing, Puhk und Spok erzählt? [...] das ist ein ungerechter Vorwurf, das ist ganz unhistorisch. Das Gegenteil ist wahr, denn die Geschichte lehrt das Gegenteil. Alles heidnische Wesen, der Götzendienst und Aberglaube der Heiden, erschien jenen christlichen Missionären als ein Teufelswerk, war in ihren Augen ein Greuel und Scheuel. Wie sollten sie den zu bekehrenden und vom heidnischen Aberglauben zu entwöhnenden Letten und Esten auch noch ihren eigenen, noch nicht völlig überwundenen Aberglauben importieren?"

s Endzelins 1908, 134.

$4 \mathrm{~Tb}$.

5 Cf. Volteris $1890,22$.

6 So Afanasjev 3, 748: Иия Овсень (Авсень, Говсень, Усень) можеть 
Argumente als unbegründet ab. Sie alle hätten sich geirrt, wenn sie das russische und lettische Wort mit der Wurzel aus- in Verbindung brachten. Statt dessen beruft er sich auf verschiedene andere russische Autoren, in erster Linie auf Markov, der davon überzeugt ist, daß der lettische Name Ūsinšs aus dem Russischen entlehnt ist. Dort seien ähnliche Wörter овсень, усень, маусень, авсень и. а. vorhanden ${ }^{1}$. Die ersten drei seien bereits in Schriften des 17. Jhs. anzutreffen und würden als Refrains in verschiedenen Liedern benutzt ${ }^{2}$.

Endzelīns glaubt, wenn er sich auf die erwähnten russischen Forscher beruft, daß von den verschiedenen russischen Wörtern dieses Typs die

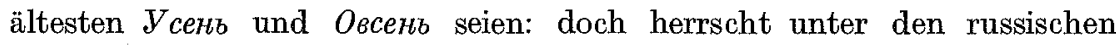
Forschern selbst weder über den einen noch den anderen dieser Namen Einmütigkeit ${ }^{3}$. Endzelīns war daher gezwungen, die Frage offen zu lassen und

быть вовведено къ тому-же санскритскому корню ush, къ которому возводися и вышеобъясненное имя Остары; согласно съ зтимь производетвомъ, Овсень долженъ означать бога возжигающаго солнечное колесо и дарующаго свътъ міру (т. е. приводлщаго съ собой утро дня или утро года=весну)." Sо auch Famincyn 252: Свиттовая, солнечная природа Усеня —- Усеня или Авсеня проявляется и въ самомъ навваніи божества: ushasa (инд.), usha (вендс.), aurora вм'сто ausosa (латин.), auszrâ (литов.) = варя; Ausca (жмуд.) - богиня лучей восходящаго или заходящаго солнта, Usil (этрус.), Ausel (сабинск.) = солнце). Чрезь замьщеніе въ Зтрусскомь и Сабинскомъ названіяхъ солнца посльдней буквы $l$ окончаніемь жь, получается Латыпскій У синь и Русскій Авсень." Cf. auch Potebnja 1865, 21 und 1884, 35ss.

1 Cf. Markov 50ss.

2 Hier nennen wir als Beispiel einen Teil eines solchen Liedes, das bei Snegirev 2, $111 \mathrm{~s}$, abgedruckt ist:

Ой Овсень! ой Овсень!
Походи, погуляй
По свяшымъ вечерамъ,
По веселымъ шеремамъ!
Ой Овсень! ов Овсеыь!
Посмошри, погляди,
Ты вкойди, посъши
Къ Филимону на дворъ.
Ой Овсень! ой Овсень!
Посмошрьлъ, поглядълъ,
Ть нашель, шы взошелъ,
Къ Филимону на дворъ!

Dasselbe Lied bringt auch Afanasjev 3, 747. Einige andere Lieder befinden sich bei Dobrovolskij 116ss und Endzelins 1908, 135s, die er von Markov und Famincyn übernommen hat.

3 So leitet Snegirev, 109s, Овсень und Авсень von Овесъ 'Hafer' ab (cf. auch Bes. tuschew Rjumin 15). Veselovskij, 107s, dagegen erkennt, daß Усень aus сёнть 'säen' entstanden ist. Beide begründen ihre Ansichten mit der russischen Tradition, daß 
zu bemerken: ,Möge sie wie auch immer von den Russen entschieden werden. Ob 'Усень' oder 'Овсень' - daran ist wohl nicht zu zweifeln, daß die Letten ihren Ūsiņš von den Russen entlehnt haben."1 Es ist leicht einzusehen, daß, wenn man darauf verzichtet, dem russischen Wort eine begründete und sinnvolle Erklärung zu geben, eine solche auch für den lettischen Namen fehlt, sofern er von den Letten übernommen worden ist. Man muß feststellen, daß sich die Hypothese des Endzelīns dann allein auf die phonetische Ähnlichkeit dieser Wörter stützt.

Der russische Refrain ist auch sonst sehr schwer mit dem lettischen Ũsiňš in Verbindung zu bringen. Er ist in Liedern anzutreffen, die anläßlich der Erwartung des Neuen Jahres gesungen wurden, also zu einer anderen Jahreszeit als bei den Letten ${ }^{2}$. Man darf auch nicht die geographischen Hindernisse vergessen.

Auniņ: lehnt auch diese Hypothese von Endzelīns in seiner letzten Arbeit ron $1913 \mathrm{ab}$, indem er darauf hinweist, daß es nicht um die Entlehnung des Namens aus dem Russischen oder Deutschen gehe, sondern das Problem sei ein ganz anderes, nämlich, ,ist der Ūsin-Mythus bei den Letten eine Entlehnung aus dem Russischen oder ein uralter selbständiger Besitz des lettischen Volkes aus jener vorgeschichtlichen Zeit, da alle die sogenannten indogermanischen Volksstämme noch ein ungeteiltes Ganzes bildeten?"'3 Man kann über die letzten Worte dieses Satzes ver'schiedener

die Leute, in Erwartung des Neuen Jahres, von Haus zu Haus gehen und die Einwohner mit Haferkörnern bestreuen, die man später zusammenliest und der Frühjahrssaat beimischt. Potebnja wiederum hat bemerkt, daß in den großrussischen Gebieten, wo man die Lieder mit dem genannten Refrain kennt, die erwähnten Neujahrstraditionen unbekannt gewesen sind. Endzelins kommt hier zu Hilfe mit dem Hinweis, daß dort eine solche Tradition vielleicht dennoch vorhanden gewesen sei, die aber verschwunden ist, und beruft sich besonders auf Dobrovolskij. Die hier erwähnten Wissenschaftler sind sich auch weder darüber klar, was diese verschiedenen Formen bedeuten, noch welche von ihnen die ältere sein könnte. Aničkov, 316, hält У сень dagegen für die ältere Form. Ưber diese Diskussion haben Endzelīns 1908, 137, und Auninš 1905, 3ss, eingehend referiert. Cf. auch Markov, besonders 55; Ljapunov 148.

1 Endzelins 1908, 138.

2 U'm den großrussischen Refrain, der in Erwartung des Neuen Jahres gesungen wurde, mit dem Frühjahr in Zusammenhang bringen zu können, wird sogar behauptet, daß diese russischen Lieder im Frühjahr gesungen worden seien und daß der Frühjahrmonat Усень genannt wurde (Markov 60 ss).

3 Auniñs $1913,14 \mathrm{~s}$. ,Es ist doch fraglos gewiß, daß die lettische Sprache eine selbstständige Sprache ist, wenn wir auch zahlreiche Wörter in ihr finden, die aus den Sprachen der Völker herübergenommen sind, mit denen die Letten im Verkehr gestanden haben. Aber alle, mit anderen verwandten indogermanische Sprachen gemeinsame Wurzeln brauchen nicht immer Entlehnungen zu sein. So sind fraglos zilweks, galwa, roka etc. uralt selbstständige lettische Wörter und keine Entlehnungen. Wenn auch das lettische wahrit und das Rußisch варить offenbar identisch sind, so 
Meinung sein, aber der im ersten Teil ausgedrückte Gedanke ist jedenfalls richtig. Es geht nicht um die Frage der lettischen Herkunft des Namens, sondern um - wie Auniņš sagt — den Ūsinšs-Mythus selbst, d. h. die religiösen Vorstellungen.

Endzelins hat seine Hypothese niemals direkt widerrufen, aber daß er seine Ansicht korrigiert hat, kann man annehmen. Er gab das von Mīlenbachs zusammengestellte Wörterbuch (ME) heraus und hat darin beim Namen Usiṇš die Bemerkung gemacht: ,In diesem Fall muß die Ansicht

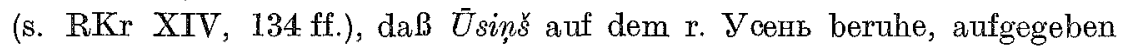
werden. "1 Der Hinweis auf die Stelle, wo diese Ansicht ausgesprochen wird, ist in Wirklichlzeit ein Hinweis auf seine eigene Arbeit von 1908. Damit hat Endzelīns seine Ansicht von der Entlehnung des Namens Ūsiņš aus dem Russischen fallen gelassen. Man kann auch das Datum feststellen, denn der Band, in dem diese seine Worte abgedruckt sind, erschien im Jahre 1932.

1.3. Mit dem Problem des Ūsiņš hat sich auch S̆mits mehrfach beschäftigt. Bereits im Jahre 1903 äußerte er die Meinung, daß die Lieder über Ūsiṇš, die Auniņš veröffentlicht hatte, Fälschungen seien, daher sei auch Ūsiņš als Pseudogott anzusehen ${ }^{2}$. Zu dieser Frage kehrte Śmits im Jahre 1911 zurück, als er wiederum die Liedertexte von Auniņ̌ als Fälschungen abtat und folgerte: ,Wenn man alles dieses erwägt, muß man unwillkürlich bei den Zweifeln bleiben, ob Ūsinš wirklich in alten und echten Volksliedern vorkommt. Selbst wenn Usiņš in diese Lieder schon in alten Zeiten Eingang gefunden hätte, könnten wir ihn immer noch nicht als Pferdegott bezeichnen. Auch wenn wir alle diese Zweifel fallen lassen würden, wäre Ūsiņš, als ein entlehnter Gott, nicht zu der alten lettischen Mythologie zu rechnen. "s Am Schluß der Abhandlung hat Šmits in klaren Worten mitgeteilt, woher

wird man doch nicht sagen dürfen, die Letten hätten das Kochen von den Russen gelernt; oder wenn das lettische strihdus, strihdèt mit dem deutschen Streit und streiten eine gemeinsame Wurzel hat, so ergiebt sich daraus noch nicht, daß die Letten erst von den Deutschen das Streiten gelernt haben. Ist die lettische Sprache kein bloßes Conglomerat von Entlehnungen aus anderen Sprachen, sondern eine selbstständige Sprache und haben die Letten zu allen Zeiten, seitdem sie sich losgetrennt haben von den ihnen stammverwandten Völkern, ihre eigene Sprache besessen, dann haben sie auch ihre eigene Gedankenwelt gehabt, auch eigene Vorstellungen und Ideen in Betreff der unsichtbaren, überweltlichon Dinge besessen. Man hat bisher noch kein vollkommen religionsloses Volk gefunden. Willkürliche Geschichtskonstruktion mag das behaupten" (ib. 15).

1 Me 4, 410. Die Abkürzung „RKr XIV“" entspricht Endzelīns 1908.

2 Šmits 1903, 172: „So kann auch Herr Auniṇš nicht deshalb verachtet werden, weil er diese gefälschten Lieder aufgenommen hat, wohl aber die Fälscher, die sich diese Lieder ausgedacht haben."

3 Śmits 1911, 20. 
Usiņš entlehnt worden sei: „Weiter muß ich mich der Ansicht Endzelīns' anschließen, daß Usinš von dem russischen 'У сень' entlehnt worden ist." Aber daß Šmits seiner Ansicht und Anlehnung an Endzelins nicht ganz sicher war, zeigt eine späte Aussage von ihm aus dem Jahre 1926, in der er neben der Entlehnung aus dem Russischen weiterhin die Möglichkeit zuläßt, daß auch eine Entlehnung vom deutschen hûsing erfolgt sein könnte, welche Endzelins selbst schon aufgegeben hatte. Smits hatte sich anschei-

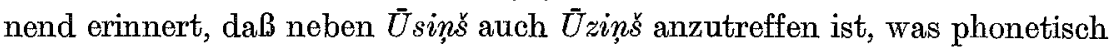
an das deutsche Wort anklingt. Für Smits stellte sich das Bild so dar, daß es ,,am Anfang zwei Gottheiten gegeben habe: Usinšs, der sich vom russischen 'У сень' herleitet, und Uziṇš, der vom deutschen 'Husing' entlehnt ist $^{\prime \prime 2}$. Das ist ein hoffnungsloser Versuch, mit Hilfe der Phonetik und etymologischer Zusammenhänge das Wesen einer Gottheit - in diesem Fall sogar zweier Gottheiten - zu erklären. Zugleich hat Smits die einfache Tatsache vergessen, daß die Letten ein Wort üzas 'Hosen' besitzen, das

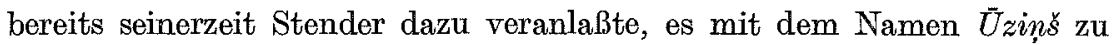
verbinden ${ }^{3}$. Es scheint, daß Smits noch eine Weile an seiner Ansicht festgehalten hat, bis Endzelins in aller Stille seine Meinung über die Entlehnung des Namens aus anderen Sprachen änderte.

1.4. Die letzte Phase der Auffassungen von Endzelins und Smits endete mit neuen Behauptungen. Ebenso wie im Falle der beiden zuvor erwähnten Hypothesen stützten sich diese auf etymologische Vermutungen. Zuerst hatte sich Endzelinns, indem er seine deutschen und russischen Hypothesen aufgab, an den in der Abhandlung von Auniņš im Jahre 1881 abgedruckten Brief von dessen Vorgänger Müthel an Ulmann erinnert ${ }^{4}$, in dem dieser schreibt, daß der Name Ūsiņš von üsas 'Schnurrbart' herstamme, wie man sich den hl. Georg mit einem solchen versehen vorstelle. Endzelīns hat sich, darauf stützend, sehr vorsichtig ausgedrückt und nur eine solche Möglichkeit zugelassen: „Und falls die Letten den heiligen Georg mit einem Schnurrbart dargestellt gesehen haben [...], so dürfte J. Müthel Recht behalten, wenn er [...] U্Usinss ( $\bar{U}$ senis, $\bar{U}$ sainis) als den 'Schnurrbärtigen' deutet." Endzelīns konnte das nur unter Zweifeln aussprechen, da das Wort üsas einen fallenden Akzent aufweist. Das schafft neue Schwierigkeiten, sofern man diese Erklärung Müthels annehmen wollte ${ }^{6}$.

\footnotetext{
1 Ib. 22. Cf. auch 1926, $22 . \quad 2$ Šmits 1926, $43 \mathrm{~s}$.

3 Cf. ME 4, 411, unter Üzinš; auch weiter S. 31 .

4 Cf. weiter S. $31 . \quad 5$ ME $4,410$.

6 Diese Schwierigkeiten sucht Endzelins zu überwinden, indem er sagt: „,[...] so

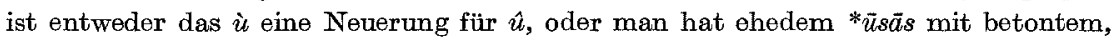
akutiertem $\bar{u}$ neben $* \bar{U}$ sinis mit betontem $\bar{\imath}$ gesprochen ${ }^{c k}$ (ib.). Wie diese Worte zeigen, ist Endzelīns gezwungen, sich mit unbewiesenen Hypothesen zu behelfen.
} 
Wie wir gesehen haben, beharrte Smits noch im Jahre 1926 auf der Auffassung der Entlehnung des Namens Ūsiňš aus anderen Sprachen. Aber Ende der dreißiger Jahre, als ihm die Meinungsänderung von Endzelins bekannt wurde, schloß er sich diesem wiederum an, ebenso wie er das vorher getan hatte, und hielt sich an dessen Ansichten. Zum letzten Male kurz vor seinem Tode hat Smits seine wechselhaften Auffassungen wie folgt zusammengefaßt: „Wir wissen auch nicht ganz genau, wie dieser Usinš bei uns entstanden ist. Früher dachten wir, daß er von den Russen entlehnt worden sei, aber ein russischer Wissenschaftler meint, daß die Russen ihn eher von den Letten geerbt haben könnten. Darin werden sich jedoch die Wissenschaftler wohl einig sein, daß er sich weder in der russischen noch in der lettischen Sprache wie ein alter und echter Volksname anhört. Wohl schon im 17. Jh. haben die Jesuiten Ūsinšs (Usching) bei den Lettgallern festgestellt, aber im Volksmund ist er nur in der Verkleinerungsform und in zwei Varianten: Ūsiņš und Ũziňš, vorhanden. Die Sammler der Volksmythen bezeichnen ihn sowohl als Pferdegott als auch als Gott der Bienen. Ein anderer will selbst diese Begriffe trennen und hält Üsins für den Pferdegott, $\bar{U}$ zinšs jedoch für den Bienengott. Hier gibt es so viele Beobachtungen und Überlegungen, daß man schwer zu wirklicher Klarheit gelangt. Man wird wohl die Hypothese von Pastor Müthel wieder zu Ehren kommen lassen müssen, die dieser bereits 1870 an Bischof Ulmann schrieb, daß Ūsiņš wohl aus üsainis 'der Schnurrbärtige' entstanden sei, wie man den hl. Georg genannt haben mochte [...]. Dieser Hypothese neigt auch J. Endzelins zu [...]. Da Ūsiņš gewöhnlich zu Georgi verehrt worden ist und Ūsiņš auch in einigen Traditionen nicht besonders von Georg unterschieden wird, wird man Müthel Recht geben müssen."1

Diese Ansicht, daß Ūsinšs seinen Namen von der Gestalt des bärtigen hl. Georg bekommen und damit auch seinen Platz in der lettischen Religion. und Folklore erhalten habe, ist demnach mit der Aussage Müthels zu verbinden, der diese Auffassung als erster geäußert hat. Endzelins ist erst nach einigen erfolglosen Versuchen, seine Ansicht zu begründen, zur Hypothese von Müthel zurückgekehrt. Die Rolle von Smits bei der Lösung des Problems Usinšs ist gering, da er lediglich Endzelīns in dessen Spuren gefolgt ist.

1.5. Kurz erwähnt werden müssen auch Aussagen über Ūsiňš, die ganz sporadisch anzutreffen sind, ohne daß sie eine weitere Begründung erfahren hätten. Sie bezeugen lediglich eine weitere Überlieferung der einen oder anderen der genannten Hypothesen mit der Tendenz sich zu festigen. In mehreren Arbeiten, die umfassende Übersichten über die Religion der

I Pasakas 1962 s 13, 319. 
Balten geben, sind sie wiederholt anzutreffen, so daß man sie nicht übergehen kann.

Es scheint, daß man eine Übersicht über diese verstreuten Ansichten am besten gewinnen kann, wenn man sie in der chronologischen Abfolge ihrer Entstehung betrachtet. Noch Ende des vorigen Jhs. haben sich drei andere Autoren zu diesen Fragen geäuBert. Das waren Kiparts, Volteris und Lautenbachs. Doch stehen alle drei unter dem direkten Einfluß von Auniņs.

1.5.1. Das Problem Ūsinš wurde international bekannt durch einen Beitrag von Kiparts, der bereits 1887 in Fortsetzungen in der damals sehr bedeutenden Zeitschrift „Revue des traditions populaires" zu erscheinen begann. So ist die 1892 gedruckte Fortsetzung direkt dem Problem Usinšs gewidment. Kiiparts stützt sich hauptsächlich auf die Aussagen von Auniņš und auf dessen Texte. Er hat einen Teil (12) von Auniņšs Texten in lettischer Sprache mit französischer Übersetzung abgedruckt. Einen Text hat er auch aus den inzwischen erschienenen Texten von Volteris übernommen. Er trägt dazu nichts Neues bei, sondern wiederholt die etymologisch begründete Argumentation von Auniņš: „,Aux divinités de la lumière, chez les Latviens, appartient incontestablement aussi Uhsin (l'Oucigne), quoiqu'à travers les siècles il soit devenu simple patron des chevaux. L'Uhsin ou Ushin latvien correspond en tous points à l'Ushas de l'ancienne religion des Indes, à laquelle sont consacrées une grande partie des hymnes du Rig-Véda."1 $\mathrm{Er}$ geht sogar noch weiter und erkennt, daß der Kult des lettischen Ūsinš auch in den Traditionen der Varäger bezeugt sei².

1.5.2. Volteris selbst hat Material in Latgale gesammelt und mehrere dort gefundene Lieder, in denen der Name Usinšs erwähnt wird, veröffentlicht. Auf den Quellenwert dieser Lieder werden wir später zu sprechen kommen und eine Quellenanalyse vornehmen. Das gesammelte Material und die Bekanntschaft mit den von Auniņš geäußerten Ansichten haben Volteris davon überzeugt, daß diese richtig sind. Er hat zwar in Latgale die Form Jeuseņš angetroffen, sie aber richtig als mundartliche Eigentümlichkeit erklärt ${ }^{3}$. Im Endergebnis erkennt er unter Berufung nicht nur auf Auniņš, sondern auch auf Potebnja ${ }^{4}$, daß der Name $\bar{U}$ siņš etymologisch mit der Wurzel us- zu verbinden ist und seinem Wesen nach den - aus derselben Wurzel abgeleiteten - religiösen Vorstellungen anderer indoeuropäischer Völker entspricht ${ }^{5}$.

\footnotetext{
1 Kiparts 260. Dort folgt eine von Auniņš übernommene Übersicht über die Etymologie des Wortes uṣas in den verschiedenen indoeuropäischen Sprachen.

2 Cf. ib. 554. $\quad 3$ Cf. Volteris 1890, 20. $\quad 4$ Potebnja 1865, 21s.

5 Volteris 1890, 21: ,Әтимологін всвхъ шяти формь ясная: всъ онъ произведены оть корня us-, латинск. urere, лит.-латыш. aust, aušti свътать. Изъ санскритск. сюда принадлежкть ušasa sаря, usar утро."
} 
1.5.3. Lautenbachs veröffentlichte im Jahre 1901 seine erste Arbeit, die einen umfassenden Einblick in die Religion der Letten zu geben versuchte. Darin erwähnt er auch wiederholt Ūsins.s. Er hat keine selbständige Quellenanalyse geliefert, sondern sich mit einem informativen, aus früheren Forschungen gewonnenen Ergebnis begnügt. Lautenbachs bezeichnet Ūsinšs wiederholt als Lichtgott ${ }^{1}$. Als solchem werden ihm positive Eigenschaften zugeschrieben, sowohl als Erwecker der Natur im Frühjahr, als auch als Spender von Wohlstand². Ūsiņ̌̌ überwindet die dunklen Mächte: den Winter und die Nacht. Lautenbachs stellt sich dann vor, daß diesem Gott zu Ehren großartige Frühlingsfeste mit Opfern veranstaltet wurden. Es scheint, daß er sich nicht direkt auf die von Aunin̄š und dessen Vorgängern intensiv gesuchten und behaupteten etymologischen Zusammenhänge beruft.

Man muß feststellen, daß keiner dieser drei genannten Autoren etwas Neues zur Lösung des Problems Ūsiņš beigetragen hat. Ihr Verdienst ist es lediglich, mit ihren in französischer, deutscher und russischer Sprache veröffentlichten Arbeiten dazu verholfen zu haben, die Aufmerksamkeit der internationalen Forschung auf das Problem des Ūsinš̌ zu richten. Man kann sagen, daß sich damit Ende des 19. Jhs. die Ansicht begründet und großenteils auch gefestigt hat, daß Ūsiņš etymologisch mit der indoeuropäischen Sprachwurzel aus- verbunden werden muß und daß er mit anderen, diesen Völkern bekannten Lichtgottheiten verwandt ist.

1.5.4. Wie wir gesehen haben, erhoben sich schon zu Beginn dieses Jahrhunderts scharfe Einwände gegen die Ansichten von Auniņ̌s. Endzelīns und Smits haben letztere wiederholt abgelehnt, waren aber gezwungen, ihre Auffassungen zu ändern. Im ganzen kann man sagen, daß die Ansichten dieser beiden Gruppen vorherrschend waren, und das sind sie bis auf unsere Tage geblieben. Man kann lediglich von gewissen Modifikationen bei ihrer Verbreitung sprechen. An erster Stelle ist hier Adamoviǒs zu nennen. In seiner Übersicht der Religion der alten Letten hat er auch Ūsiņš erwähnt. Er folgt der Linie von Smits und erkennt in Usiņš einen Pferdepatron ${ }^{3}$. In

1 Cf. Lautenbachs 1901, 175, 195, 230.

2 Ib. 230: ,Dann hat man alljährlich im Frühling, den 23. April, eine Usin̄-Feier begangen zu Ehren des Lichtgottes Usin, dessen Söhne Morgenrot und Abendrot, ja Sonne und Mond, sind, der die ganze Welt erwärmt, der im Frühling mächtig dahergesprengt kommt auf einem Rosse von Stein, Schnee und Eis zermalmend, - der Wälder und Wiesen mit herrlichem Grün bekleidet und nicht bloß den Pferden, sondern allen lebenden Wesen die zur Lebenserhaltung nötige Nahrung schafft und spendet. An diesem Festtage sind Speise- und Trankopfer, bestehend aus Fleisch, Brod und Bier dargebracht worden; auch hat an diesem Usintage ein solennes Festmahl stattgefunden $[\ldots]]^{\text {c: }}$

3 Adamovičs 1937, 63, 71. 
bezug auf den Namen von $\bar{U} \operatorname{sing} s$ gerät er in Unklarheiten. Einerseits beruft er sich auf Smits, der den Namen für eine Entlehnung aus dem Russischen hält, aber gleichzeitig äußert er sich selbst nicht über die Richtigkeit dieser Ansicht von Smits. Andererseits möchte er den Gedankengang von Smits nicht recht übernehmen. „Und selbst wenn der Name Ūsiņš eine Entlehnung vom russischen $\mathrm{Y}$ сень darstellt, beweist das noch nicht, daß die alten Letten nicht einen Pferdepatron mit einer eigenen Bezeichnung gehabt hätten." ${ }^{1}$ Doch hat sich Adamovičs selbst dazu nicht näher geäußert, weder zu der Bezeichnung dieses Pferdegottes, noch hat er dessen Wesen im Unterschied zu Ūsiņš weiter charakterisiert. Das ist verständlich, da er keinerlei Forschungen auf diesem Gebiet vorgenommen hat. Dennoch hat Adamovičs mit seiner Behauptung dazu beigetragen, die Ansicht über Ūsiṇš als einen Pferdegott weiter zu bestärken, wie wir noch spät, im Jahre 1969, lesen können ${ }^{2}$. Daneben hat sich bis auf unsere Tage auch noch die Behauptung erhalten, daß Ūinšs ein Bienengott $\mathrm{se}^{3}$, obwohl gewöhnlich dafür gar keine Beweise geliefert werden.

1.6. Zusammenfassend kann man sagen, erstens, daß sich in diesem Jahrhundert die Ansicht gefestigt hat, Ūsinšs sei ein Pferdegott, während die Meinungen über den Ursprung seines Namens geteilt sind. Zweitens bestehen vereinzelt auch Behauptungen über Ūsinšs als Bienengott. Drittens ist die von Auniņš begründete und später von Ķiparts, Volteris und Lautenbachs gestützte Auffassung von Usiňš als einem Lichtgott so gut wie vergessen ${ }^{4}$. Erst nach Einsichtnahme in die Quellen und deren inhaltlicher Analyse werden wir an eine kritische Bewertung dieser Ansichten herangehen können.

1 Ib. 64.

2 Cf. Balevies 208. Wir erwähnen hier noch einige Vertreter dieser Auffassung, ohne daß sie neue oder zusätzliche Begründungen geboten hätten: Arājs 1957, 635; id. 1957a, 800; Ligers 181; Straubergs 1934, 21742, 21750.

s Cf. Ozols 1955, 432; LE 3, 2537;

4 In Wirklichkeit hat sich auf Grund der Ansichten von Auniñš eine ähnliche Überzeugung entwickelt, bestimmt durch den Wunsch, die Religion der alten Letten zu ernevern. Doch fehlt den Arbeiten dieser Autoren das kritische Bewußtsein, und in ihnen werden teilweise begründete Behaiuptungen mit in freier Phantasie gebildeten Kombinationen gemischt. Sie haben zur Lösung des Problems nichts Neues und Bedeutendes beigetragen, daher ist eine Erörterung darüber hier nicht am Platz. Wir möchten hier nur ein Beispiel aus der Arbeit des Begründers dieser Bewegung, Brastinšs, 1966, 59, erwähnen, wo behauptet wird, daß ,auch der lettische Üsinš Gottessohn genannt“* wird. (Cf. auch id. 1929, 50). Es gibt keine Quelle, die das bezeugen würde, daher ist diese Behauptung nicht belegt. Thm folgten Brastiņš, 1956, 73ss; Audriňs 33ss; Audzis $86 \mathrm{ss}$, und andere Vertreter dieser Bewegung. 